\title{
Improving Vector Quantization of Satellite Images Through the Application of Bi-orthogonal Wavelets
}

\author{
Anthony Tung-Shuen Ho, Tao Yu, Siu-Chung Tam, \\ Siong-Chai Tan and Lian-Teck Yap \\ Nanyang Technological University, \\ School of Electrical and Electronic Engineering, Nanyang Avenue, Singapore 639798 \\ Tel: (65) -790-6373 / Fax: (65) -792-0415 / E-mail: etsho@ntu.edu.sg
}

\begin{abstract}
In image coding applications, vector quantization (VQ) has widely been accepted as an effective compression method for digital images. However, its-effectiveness depends greatly on the matching of the VQ elements with the image data values. Prior to the-VQprocess, orthogonal transforms such as a DCT or FFT are often used to convert an image in the spatial domain to transform coefficients in the frequency domain. In this paper, instead of orthogonal transform coeffieients, bi-orthogonal wavelet filters are used to transform a satellite image to the scale-frequency domain:

The wavelet coefficients are vector quantized and their statistical features are analyzed in details such as computational complexity and performance efficiency. Some examples will be shown in the presentation to illustrate the advantage of using bi-orthogonal wavelets that can hefp to improve the matching of VQ elements to the image data values. Through matching the image values, it can be shown that fewer VQ efements would be required to represent the image while maintaining the image quality. Improving the VQ statistical features will in turn increase the compression ratios of image compression of satellite images.
\end{abstract}

\section{INTRODUCTION}

The main objective of applying digital image compression techniques is to minimize the number of bits required to represent an image, while maintaining acceptable image quality. Véctor quantization (VQ) has been proven to be an efficient method to code speech, image and video data. The application of satellite remote sensing has led to the tremendous growth in the transmission and storage of digital images. The needs for efficient methods of data compression become more and more important. For the past ten years or so, the resolutions of remote sensing satellites have increased significantly that made data compression even more necessary. In general, satellite images contain less redundancy information compared to normal images. It is therefore not easy to obtain high compression ratios. Vector quantization (VQ) has been commonly employed in speech and image signal processing applications. However, the undesirable blocking effects of VQ decoded images tend to limit the application of VQ coding in satellite image compression.
The statistical features of an image will influence the overall performance of vector quantization. Many image compression schemes incorporate a combination of orthogonal transforms such as DCT or FFT, and vector quantization of the transform coefficients based on an optimum codebook design. Nevertheless, the statistical features of an image are other important factors that could affect the efficiency of the codebook, as well as the quality of the reconstructed images.

Recently, the subject of "Wavelet analysis" has attracted much attention from both mathematicians and engineers alike. Wavelet theory has been applied in many fields and applications, such as image analysis, communication systems, biomedical imaging, radar, and control systems. With wavelet transforms, the original image is first decomposed at different scales or sub-bands. This transformation also changes the statistical features of the image and in turn, could improve the performance of vector quantization.

\section{WAVELET PRSENTATION OF IMAGE}

Wavelet transform is an orthogonal (or bi-orthogonal) transform. Compared to a normal FFT or DCT orthogonal transform, wavelet transform is a new time-frequency twodimension signal analysis method. Haar described the first wavelet basis in 1910 known now as the Haar wavelet basis. However, this wavelet does not form a continuous basis. In the $1980 \mathrm{~s}$, researchers such as Coheb, Coifman, Daubechies $^{[4]}$, Mallat ${ }^{[2]}$ and Meyer performed a lot of pioneering work in wavelets, especially in multi-resolution and fast wavelet transforms. As a result, wavelet transform began to find more and more applications in digital signal and image processing.

Although the theoretical basis of orthogonal analysis is very sound, it is not simple to derive an orthogonal wavelet basis. Normally, the basis of orthogonal wavelet transform is neither compact support nor continuous. This means it is not a straightforward task to design linear FIR filters based on orthogonal wavelets. Moreover, the application of wavelet transforms in image processing requires linear phase FIR filters. Implementation of FIR filters is based on the use of bi-orthogonal wavelet. Bi-orthogonal wavelets are based on two multi-resolution analyses: $\left\{V_{m}\right\},\left\{W_{m}\right\}$ and $\left\{\widetilde{V}_{m}\right\},\left\{\tilde{W}_{m}\right\}$. They satisfy the following conditions:

$$
V_{m} \perp \tilde{W}_{m}, \tilde{V}_{m} \perp W_{m}
$$


And their Z-transforms satisfy ${ }^{[2]}$ :

$$
|H(z) \| \widetilde{H}(z)|+|H(-z)||\widetilde{H}(-z)|=1 \quad(|z|=1)
$$

$\mathrm{Yu}$ et al ${ }^{[1]}$ have designed several kinds of wavelets and FIR filters through B-splines. These filter banks all have the following important characteristics:

- Satisfy bi-wavelet analysis conditions;

- Bases are compact support and continuous;

- High efficiency;

- Linear phase;

- Signal can be reconstructed exactly.

\section{A. Computation}

Compared to FFT and DCT, the computation of wavelet transform does not depend on the size of the signal. Now let us compare the computation of the different transforms.

It is known that ${ }^{[5]}$ :

- $\quad \mathrm{N}$-point FFT requires $N \log _{2} N$ complex multiplications.

- N-point DCT requires $\left(N \log _{2} N-N+2\right) / 4$ complex multiplications.

For wavelet transforms, the formula would be quite different, as its computation is not dependent on the number of points. For $\mathrm{m}=2$ and $\mathrm{m}^{\prime}=2$ bi-orthogonal wavelet transforms, its average computation is 2.5 real multiplications. But if all the parameters of FIR are considered to be the power of (1/2), then multiplications can be converted to bit shift if performed on a computer or a DSP chip. This bit shift operation leads to a fast bi-orthogonal wavelet transform. Its average computation is 1 real multiplication and 2.5 real additions, as presented in table 1 .

Table1. Comparison of Number of Multiplications For Different Methods

\begin{tabular}{|c|c|c|c|c|}
\hline N & 4 & 8 & 16 & 32 \\
\hline FFT & 32 & 96 & 256 & 640 \\
\hline DCT & 6 & 18 & 50 & 130 \\
\hline WL & 10 & 20 & 40 & 80 \\
\hline FWL & 4 & 8 & 16 & 32 \\
\hline
\end{tabular}

Consider the different values of $\mathrm{N}$ in Table 1, which lists the computation efficiency of FFT, DCT and Wavelet, high efficiency can be obtained by the Fast Wavelet Transform with $\mathrm{N}>8$. With increasing $\mathrm{N}$, the advantage of higher efficiency for the fast wavelet transform becomes more and more apparent.

Wavelet transform is thus a high efficiency and low computationally intensive method for transforming a signal in the time domain to the time-frequency domain. Its computation is much lower than the FFT or DCT. The key advantage of the wavelet transform is that its number of computations per point remains constant. This means that the entire signal may be wavelet transformed without the need use sub-blocks to reduce computation effort, as would be required for the FFT or DCT transform, resulting in blocking effects.

\section{B. Statistical Features}

A wavelet transform will modify the statistical features of a signal. Typically, a wavelet transform includes a filter pair: a high-pass filter and a low-pass filter. In this paper, the biorthogonal wavelet FIR filter is used for image transformation. The initial iteration of the bi-orthogonal wavelet FIR filter will act on the satellite test image, decomposing it into a coarse and three detail components. The next iteration on the coarse component of the previous iteration decomposes it further into coarse and detail components. After $\mathrm{N}$ decomposition iterations, $3 \mathrm{~N}$ detail components and one coarse component of the original image are yielded. The coarse component of the decomposition can be regarded as a low-pass filtered sub-image and the detail components as the high-pass filtered sub-image of the original signal. The pixel values in these components are always small and lowly correlated.

For vector quantization, the data statistics are very important. It is expected that the statistical features between a training image and a test image may be very similar. In general, however, different satellite images would have different statistical features. Therefore, it is almost impossible to get a common codebook to satisfy all of these images. DCT or FFT is often used to transform data from the time domain to the frequency domain in order to get better results, but the results are not satisfactory due to computation and blocking effects. As wavelet transform can also transform an image from the time-domain into the frequency domain, it offers an alternative opportunity to compress an image at different scales and sub-bands.

Let us examine two images; figure 1 shows the statistical features of an image of a city scene and the detail components after wavelet transform; figure 2 shows the corresponding image of a lake scene. The statistics of these images are very different. But after wavelet transform, their detail components become very similar.

\section{SATELLITE IMAGE COMPRESSION}

After a wavelet transform, the statistics of an image would be changed. Applying several iterations of a high-pass wavelet filter would cause the pixel values of the detail components to become smaller and less dependent on the original image, benefiting the vector quantization process. On the other hand, the coarse component becomes highly correlated and is not easy to quantize.

Vector Quantization is a good method to compress images to very low bit rates. The principal objective of any digital image compression technique is to reduce the number of bits required to represent an image, while maintaining acceptable image quality. The block size for vector quantization and the size of codebook are the two important parameters that have a major influence on the quality of vector quantization. If the block size increases, a higher compression ratio can be 
obtained for certain vectors of some images. For other images, the quality of vectors would be somewhat poorer. In this paper, after comparing the results of different sizes of vectors and codebooks, a vector size of 32 by 32 and a codebook of size 128 were chosen. For the coarse component, the ADPCM method was used. The wavelet transform was applied only once in each of the two dimensions to generate one coarse component and three detail components. Followed by vector quantization on the detail components and ADPCM on the coarse component. A reconstrueted image with $P S N R=28.65 \mathrm{~dB}$ was subsequently obtained. To test the quality of the codebook, two different images were used. One is a lake scene and the other is a mountain scene. The codebook obtained through the city scene was used, the reconstructed images of these two scenes were reasonable good, with $P S N R=28.34 \mathrm{~dB}$ and $31.73 \mathrm{~dB}$, respectively.

\section{CONCLUSION}

The effects of bi-orthogonal wavelet FIR filter on the statistical features of satellite images were studied and evaluated. The computation efficiency of bi-orthogonal wavelet was also analyzed in detail. The result proves that the bi-orthogonal wavelet FIR filter pair can be used to transform an image from the time domain into the time-frequency domain with low computational overheads. Using biorthogonal wavelets can improve the matching of vector quantization elements to the image data values. In image coding applications, vector quantiżation (VQ) has been widely accepted as an effective compression method for digital images. However, its effectiveness depends greatly on the matching of the VQ elements with the image data values. Through matching the image values, it can be shown that fewer VQ elements would be required to represent the image while maintaining the image quality. Improving the VQ statistical features will in turn increase the compression ratios of image compression of satellite images.

\section{REFERENCES}

[1] T. Yu, A. T.S. Ho, et al, "A Novel Hybrid Bi-orthogonal Wavelets/ADPCM Algorithm For Very Low Bit Rate Satellite Image Compression", accepted for IGARSS'99, Hamburg, Germany.

[2] S.G.Mallat: "A Theory for Multi-resolution Signal Decomposition: The Wavelet Representation", IEEE Trans. Pattern Analysis and Machine Intelligence, Vol.11, No.7, pp.674-693, July 1989.

[3] N. B. Karayiannis, P. I. Pai and N. Zervos, "Image Compression Based on Fuzzy Algorithms for Learning Vector Quantization and Wavelet Image Decomposition", IEEE Trans. On Image Processing, Vol. 7, No. 8, pp1223-1230, August 1998.
[4] M.Antonini, M.Barlaud, P.Mathieu, and I. Daubechies," Image coding using wavelet transform", IEEE Trans. Image Process, Vol.1 pp.205-220, February 1992.

[5] K.R.Rao and P.Yip, "Discrete Cosine Transform: Algorithms, advantages, applications", Boston: Academic Press, 1990.
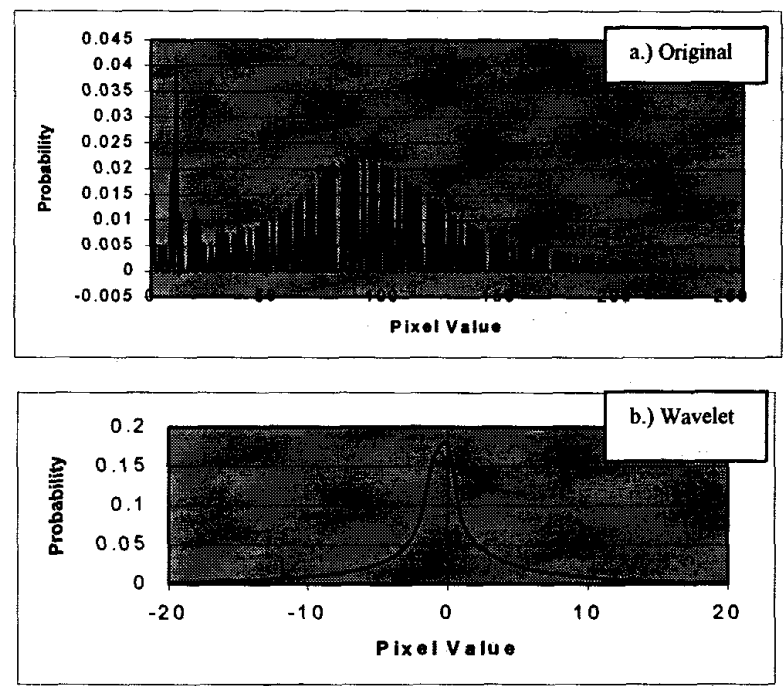

Fig1. City Scene Image and Its Wavelet Transform
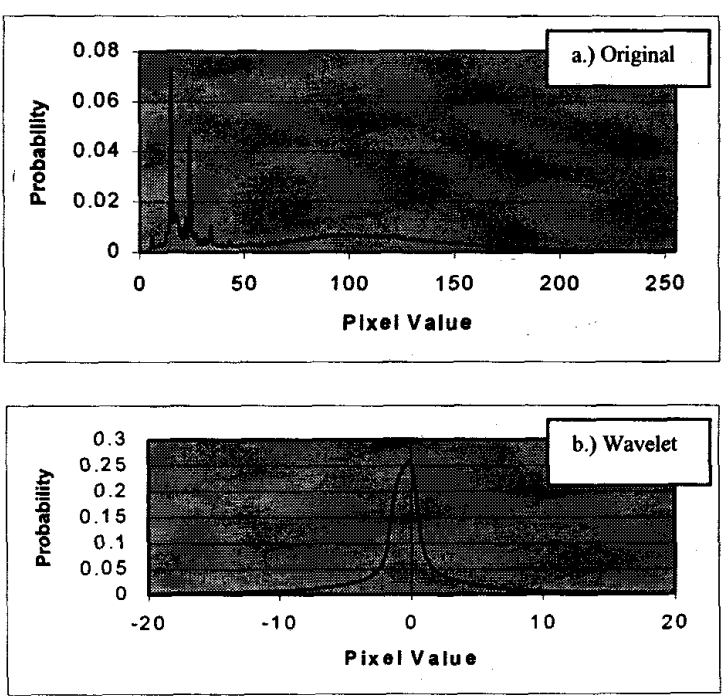

Fig2. Lake Scene Image and Its Wavelet Transform 